\title{
Evolución histórica del tratamiento de la diabetes tipo 1 en México
}

\section{Historical evolution of the treatment of type 1 diabetes in Mexico}

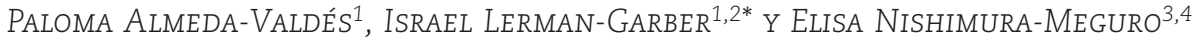

'Departamento de Endocrinología y Metabolismo, Unidad de Investigación de Enfermedades Metabólicas, Instituto Nacional de Ciencias Médicas y Nutrición Salvador Zubirán; ${ }^{2}$ Medicina Interna y Endocrinología, Centro Médico ABC; ${ }^{3}$ Endocrinología Pediátrica, Hospital de Pediatría, Centro Médico Nacional Siglo XXI, Instituto Mexicano del Seguro Social; ${ }^{4}$ ExJefatura de Endocrinología, Hospital de Pediatría, Centro Médico Nacional Siglo XXI, Instituto Mexicano del Seguro Social. Ciudad de México, México

\section{RESUMEN}

Con el advenimiento de la insulina, la diabetes mellitus tipo 1 (DM1) dejó de ser una enfermedad fatal para convertirse en una enfermedad crónica, asociada a descompensaciones agudas e importantes complicaciones microvasculares y macrovasculares. A lo largo de los años los importantes avances tecnológicos han facilitado lograr los objetivos terapéuticos con menor riesgo de hipoglucemias y reducción significativa en el riesgo de desarrollar complicaciones. Estos avances, sin embargo, aún no son accesibles a la gran mayoría de los pacientes. En este capítulo se presenta en forma breve la evolución del tratamiento de la DM1 en nuestro país y permite hacer un repaso a la historia del manejo de la DM1.

Palabras clave: Insulinoterapia. Diabetes mellitus tipo 1. Control intensivo de la diabetes. Historia de la diabetes en México.

\begin{abstract}
With the advent of insulin, type 1 diabetes (T1D) was no longer seen as a fatal disease and transformed in a chronic disease, associated to acute decompensations and chronic micro and macrovascular complications. Through the years important technologic advances have made easier to achieve therapeutic objectives with less hypoglycemic risk and significant reduction of complications development; however, these are not accessible for most patients. In this chapter the evolution of the treatment of T1D in our country is briefly described allowing to review the history of the management of T1D.
\end{abstract}

Key words: Insulin therapy. Type 1 diabetes. Intensive diabetes control. History of diabetes in Mexico.

\section{Correspondencia:}

*Israel Lerman-Garber

E-mail: israellerman@gmail.com
Fecha de recepción: 02-08-2021

Fecha de aceptación: 01-11-2021

DOI: 10.24875/RME.M21000019
Disponible en internet: 30-11-2021

RevMexEndocrinol Metab Nutr.2021;8:(SUPL.3):47-58

2462-4144 / @ 2021 Sociedad Mexicana de Nutrición y Endocrinología, AC. Publicado por Permanyer. Este es un artículo open access bajo la licencia CC BY-NC-ND (http://creativecommons.org/licenses/by-nc-nd/4.0/). 


\section{ANTECEDENTES}

La introducción de la insulina en la práctica clínica transformó rápidamente una enfermedad fatal como la diabetes mellitus tipo 1 (DM1) en una enfermedad crónico-degenerativa. Antes de los años 70 el monitoreo del paciente se realizaba con glucosurias, se utilizaban básicamente insulina $\mathrm{NPH}$ (Neutral Protamine Hagedorn) o intermedia y la insulina rápida, ambas de origen animal. En los años 80 hubo grandes avances en el desarrollo de insulinas, sus formas de administración, en alternativas para lograr un monitoreo más estrecho de la glucosa en forma ambulatoria y la hemoglobina glucosilada se empezó a utilizar para evaluar el control glucémico. En 1993 se publicaron los resultados del Diabetes Control and Complications Trial (DCCT), cuyo objetivo primordial fue probar la hipótesis del papel de la glucosa en el desarrollo de las complicaciones tardías de la diabetes ${ }^{1,2}$. El DCCT generó en EE.UU. y rápidamente en el resto del mundo el inicio en la era del manejo intensivo de la diabetes. Antes, muchos médicos consideraban que el objetivo de estar cerca de cifras normales de glucosa no se podía alcanzar y era suficiente con evitar descontrol severo o crisis hipoglucémicas y las cifras de hemoglobina glucosilada no eran menores del $9.0 \%$. Los importantes avances tecnológicos de las últimas décadas han facilitado el control intensivo y lograr los objetivos terapéuticos con menor riesgo de hipoglucemia, pero no son accesibles a la gran mayoría de los pacientes por razones fundamentalmente económicas y socioculturales. Esto ocurre en México y en la mayoría de los países. Una enfermedad como la DM1 requiere de muchos recursos y educación que no tienen la mayoría de los pacientes y sigue siendo un gran reto para todos quienes estamos involucrados en el manejo de pacientes con esta enfermedad.

\section{LA DIABETES TIPO 1 EN MÉXICO}

La diabetes en poblaciones pediátricas en México está formada por un grupo heterogéneo de pacientes con una combinación de DM1 (que aún es la que predomina) y diabetes tipo 2 (DM2) de inicio temprano, la cual se presenta muy rara vez antes de la pubertad. En nuestro país, donde hay una elevada prevalencia de sobrepeso $u$ obesidad en niños $y$ adolescentes, es común que pacientes con DM1 tengan historia familiar de DM2 y un fenotipo de resistencia a la insulina. En una publicación reciente de la cohorte del Registro Nacional de Pacientes con Diabetes Tipo 1 (RENACED-DT1) ${ }^{3}$ en población mexicana con DM1, el 44\% de los pacientes con DT1 tenían sobrepeso u obesidad. En EE.UU. el fenotipo de resistencia a la insulina está presente en el $20 \%$ de los pacientes con DM1 y esta asociación es significativamente mayor en pacientes de origen hispano o de raza negra ${ }^{4}$. No conocemos la incidencia de la DM1 en México, pero al parecer es baja comparada con otros países del mundo. En 2012 en un estudio del Instituto Mexicano del Seguro Social (IMSS) se reportó una incidencia de 6.2 por 100,000 en menores de 19 años $^{5}$. En EE.UU. la incidencia es de 23.6 por 100,000 niños, siendo significativamente menor en la población de origen hispano ${ }^{6}$.

En EE.UU. cerca del 3-4\% de la población con diabetes tiene DM1 y esto corresponde a cerca de un millón doscientas mil personas ${ }^{6}$. En México la DM1 corresponde probablemente a menos del $0.3-0.5 \%$ de todos los casos de diabetes (probablemente no más de 25,000 a 30,000 pacientes), pero comprende cerca del $15 \%$ de los casos con DM en población adulta tratada en el $3^{\text {er }}$ nivel de atención y es la patología endocrina más común en edad pediátrica ${ }^{5}$.

En México alrededor del $14 \%$ de todos los pacientes con diabetes son menores de 40 años (la gran mayoría con DM2), estos se caracterizan por requerir en forma más temprana insulina y tienen un riesgo muy alto de desarrollar complicaciones de la enfermedad $^{7}$. La DM1 de inicio tardío representa el 10\% de los casos de diabetes en población mayor de 40 años de origen anglosajón, pero es mucho menos frecuente en nuestro país ${ }^{8}$.

En EE.UU. en un año alrededor de 130 mil pacientes ingresan a un hospital con cetoacidosis diabética, la mayoría con DM1, lo que refleja que probablemente un porcentaje alto de pacientes no son atendidos en centros endocrinológicos, no tienen un manejo 
intensivo de la diabetes y no logran los objetivos terapéuticos ${ }^{9}$. En un reporte de México en el 2012 del total de egresos hospitalarios en los hospitales de la Secretaría de Salud (SSA), solamente 1,100 casos fueron episodios de cetoacidosis en pacientes con $\mathrm{DM} 1{ }^{10}$, cifra relativamente baja y acorde a la prevalencia mucho menor de DM1 en nuestro país. En países desarrollados, la cetoacidosis diabética sigue siendo la primera causa de muerte en pacientes con DM1 menores de 40 años. En México probablemente ocurre algo similar y seguramente algunos pacientes fallecen por esta causa antes de llegar al hospital y sin haberse establecido el diagnóstico de diabetes.

En EE.UU. la mortalidad en niños y adolescentes con diabetes varía sustancialmente y es significativamente mayor en pacientes de origen hispano y de raza negra derivado de las diferencias socioeconómicas y de acceso a la salud. En el paciente con DM1 de larga evolución predominan como causa de muerte la enfermedad cardiovascular y/o el daño renal crónico ${ }^{11}$. La muerte asociada a hipoglucemia es afortunadamente muy poco frecuente, aunque se considera que participa indirectamente en el 2-4\% de la mortalidad favoreciendo caídas, eventos vasculares o accidentes ${ }^{11}$. La prevalencia de complicaciones tardías de la diabetes sigue siendo elevada en nuestras instituciones de salud que atienden pacientes adultos con DM1 (ceguera, insuficiencia renal, amputaciones y problemas cardiovasculares) con reducción significativa en la expectativa de vida.

\section{EL PACIENTE PEDIÁTRICO CON DIABETES MELLITUS TIPO 1 A NIVEL INSTITUCIONAL}

En el caso del paciente pediátrico con DM1, deben considerarse características especiales de acuerdo con su grupo de edad (Tabla 1), de aquí la necesidad de contar con un subespecialista en endocrinología pediátrica (EP). Una de las principales limitantes de un manejo intensivo es la mayor variabilidad glucémica con proporciones indeseadas de tiempo en hiperglucemia o hipoglucemia, especialmente esto
Tabla 1. Características del paciente pediátrico con diabetes mellitus tipo 1

Sistema nervioso central en desarrollo y más sensible a disglucemias

Etapas con menor (lactante) y mayor (adolescencia) resistencia a insulina

Limitación para comunicarse y tomar decisiones

Habilidades y destrezas relacionadas con grupo de edad

Necesidad de un cuidador primario informal

Mayor variabilidad glucémica

Visión y objetivos a largo plazo diferentes

Desarrollo psiconeurológico

último. Se calcula que aproximadamente dos tercios de la población pediátrica con DM1 en nuestro país recibe atención médica total o parcial en alguna institución de salud pública federal o estatal, con la mayoría de los equipos de salud especializados establecidos en el 3. ${ }^{\text {er }}$ nivel de atención ${ }^{5}$. Entre 1960 y 1970 los servicios de EP del 3. ${ }^{\text {er }}$ nivel de atención de las instituciones federales de salud se fundaron a finales de esta década. La formación del subespecialista era tutorial, sin reconocimiento institucional o universitario. La insulina disponible era de origen bovino y/o porcino, esta última con estructura primaria más parecida a la insulina humana y con mayor costo. De no disponer de refrigerador, el paciente debía de resguardar la insulina en una olla de barro enterrada para mantener en lo posible la temperatura baja. La insulina basal consistía en insulina lenta e insulina NPH. A la variabilidad de la farmacocinética se agregaba la posibilidad de reacciones inmunitarias, ya sea por diferencias en su estructura primaria como por las partículas extrañas reportadas como partículas por millón, que definían la pureza del extracto. Estas reacciones se reflejaban en zonas de lipodistrofia en mayor o menor grado en aproximadamente el $40 \%$ de los casos y su búsqueda intencionada era parte de la revisión clínica. No era raro observar reacción local leve en la zona de aplicación e incluso ocasionalmente sistémica. Las zonas de lipodistrofia menos sensibles eran zonas de aplicación preferidas por el paciente, pero la farmacocinética de la insulina podía ser aún más variable por «atrapamiento» de la insulina a ese nivel, por lo que había que insistir sobre no utilizar estas zonas. Había casos con grandes necesidades de 
insulina probablemente debido a fijación a anticuerpos circulantes o incluso hipoglucemias severas de larga duración que se atribuían a liberación no previsible de la insulina acumulada en zonas de lipodistrofia o unida a anticuerpos. Las jeringas eran reutilizables, de vidrio con agujas de acero, se esterilizaban después de cada aplicación colocándolas cinco minutos en agua en ebullición y por efecto del contenido mineral del agua, finalmente el émbolo iba perdiendo su capacidad de "deslizarse», por lo que había que lavarlas en agua con vinagre. Las agujas utilizadas en ese entonces tenían una longitud y diámetro mucho mayor que lo recomendado actualmente. Al ser utilizadas en múltiples ocasiones, las agujas perdían su «filo», por lo que de no ser posible la sustitución frecuente, una piedra para afilar cuchillos o una piedra de río era parte del estuche. Lo anterior hacía que el procedimiento de carga y aplicación de insulina fuese más susceptible de error y más invasivo y el estrés generado por su aplicación afectaba la relación del niño con su familia y favorecía menor adherencia.

El control glucémico se valoraba por medio de parámetros clínicos como bienestar general, poliuria, polidipsia, crecimiento y desarrollo y la presencia de glucosurias. Los niños pequeños frecuentemente tenían enuresis nocturna, ya que la variabilidad de las insulinas disponibles y el monitoreo glucémico con glucosurias impedía ajustes más precisos incluyendo en días de enfermedad. Por lo anterior, era «habitual» que la mayoría de los pacientes tuviesen entre cuatro y seis visitas a urgencias u hospitalizaciones por año por hipoglucemias, descontrol hiperglucémico o cetoacidosis franca. No se contaba con glucagón y las glucosurias como reflejo de la glucemia promedio en las horas previas incluía la recolección y cuantificación de la diuresis con los inconvenientes esperados. En ocasiones, los pacientes bromeaban sobre sus niveles de glucosa por la cantidad de pequeñas hormigas que se reunían en la zona de recolección.

Entre 1970 y 1980 continuaba el uso de insulina de origen animal y las glucosurias para el monitoreo. El uso de insulina porcina era favorecido principalmente en pacientes con lipodistrofias importantes $y / 0$ altos requerimientos de insulina, que en algunos pacientes podrían sobrepasar las $100 \mathrm{U}$ diarias. Los servicios de
EP implementaron las clínicas de diabetes y la educación en diabetes, reconocida ya como pilar en el manejo de esta enfermedad, cobró auge en nuestro país. Fue en el ámbito institucional donde en 1970 inició la implementación de educación en diabetes como actividad formal a grupos de pacientes pediátricos con DM1 fuera del ambiente familiar y hospitalario, por medio del Campo de Adiestramiento para Diabéticos Juveniles (CADJ) . Era toda una experiencia para el equipo de salud vivir con los pacientes el día a día de su enfermedad. Había que proteger y airear los colchones por enuresis nocturna frecuente, lavar jeringas y sacar filo a las agujas, hervirlas diariamente y aplicar dosis correspondiente con base en el cuadro clínico y número de ++ de glucosurias.

A mediados de los años 70 se contó con jeringas desechables que facilitaron la carga y aplicación de insulina. Se introdujo también la medición de glucemias capilares en tiempo real por comparación visual con una escala de colores (con sesgo de valoración y efecto de las discromatopsias que pueden presentan los pacientes, asociadas con el descontrol y que entonces no conocíamos) e incluso se permitía al paciente optimizar sus recursos al utilizar medias tiras cortadas a lo largo. No era tan raro tener casos con cataratas, retinopatía o neuropatía desde etapas pediátricas.

En la década de 1980 a 1990 se inició con la determinación de hemoglobina glucosilada, nuevamente por la medicina institucional pediátrica. Inicialmente hemoglobina glucosilada total y con las limitaciones que tiene comparar la metodología «artesanal» de esos tiempos con la actual automatizada, estandarizada y limitada al tipo A1c; se reportaban en promedio del $17 \%$ con algunos reportados hasta con el $25 \%$ Las complicaciones agudas y crónicas se observaban aun en edad pediátrica y tesis de algunos residentes fueron sobre la prevalencia de estas.

La insulina humana por tecnología recombinante se introdujo en 1982, con lo cual disminuyeron las lipodistrofias y reacciones a la aplicación. La insulina basal era NPH para prácticamente todos los casos y su uso antes de la cena se acompañaba frecuentemente de hipoglucemia entre las dos y tres de la mañana. Se contaba con varias estrategias (que 
siguen vigentes hoy en día), como diferir la aplicación de NPH a antes de dormir para que el pico máximo coincidiera más con el fenómeno del amanecer, reservar alimentos (generalmente la leche por su contenido de grasas, proteínas y carbohidratos) a antes de dormir o agregar una colación nocturna. La insulina humana y las jeringas desechables, aun sin la fineza de las agujas actualmente disponibles, favorecieron la adherencia.

En esta década llegaron también los glucómetros para medición en tiempo real de la glucemia capilar. Inicialmente diseñados para contar con resultados rápidamente en el lugar de atención clínica, eran de gran tamaño y muy imprecisos. La tecnología avanzó rápidamente hasta contar con dispositivos más amigables, lo que favoreció ajustes más precisos y a corto plazo. Los glucómetros inicialmente no estaban al alcance de todos, no podían leerse en la oscuridad (para obtener la muestra del paciente dormido, había de acompañarse de una lámpara y atinar la gota en la zona correspondiente y salir inmediatamente a la luz para leerlo correctamente) y no eran tan precisos.

Los pacientes pasaron a estar en general asintomáticos, aunque persistían con hipoglucemias entre cuatro y seis veces por semana. Los eventos de hospitalización disminuyeron y se limitaron a episodios de descontrol agudo severo en pacientes en su inicio o a una minoría de pacientes identificados por dinámica familiar y factores socioeconómicos muy alterados. El control glucémico mejoró y fue evidente una disminución paulatina de la incidencia de complicaciones. Los niveles de hemoglobina glucosilada, ya $\mathrm{A} 1 \mathrm{c}(\mathrm{HbA} 1 \mathrm{c})$, promediaban el $12 \%$ para todo el grupo de pacientes pediátricos, con menores niveles en prepúberes. Durante esta década, se implementaron los cursos de adiestramiento en EP para pediatras con reconocimiento institucional (Instituto Nacional de Pediatría [INP]).

Fue en 1993 cuando se reportaron los resultados del $\mathrm{DCCT}^{1}$ que confirmaron lo que la experiencia y estudios previos sugerían: es posible lograr un mejor control glucémico (incluso en adolescentes) con esquemas de tratamiento intensivo y esto se acompaña de menor riesgo de complicaciones crónicas, pero mayor riesgo de hipoglucemias.
La insulina basal disponible continuaba siendo NPH e insulina regular como bolo, con una farmacocinética lejos de lo ideal. En la diabetes en el primer año de vida con alta sensibilidad a insulina, el uso de insulina regular estaba limitado a hiperglucemias severas y persistentes y la dificultad para cargar dosis pequeñas como $0.5 \circ 0.25 \mathrm{U}$ a concentraciones habituales obligaba al familiar a contar con una destreza más, realizar diluciones de insulina con solución salina en cada aplicación.

Aparecieron las microinfusoras en nuestro país y en varias instituciones se introdujeron como parte de protocolos de investigación o apoyos de la industria. Incluso en 1992, se realizó un CADJ para adolescentes con DM1 candidatos a uso de microinfusora. Fue toda una experiencia para el equipo médico y los pacientes se adaptaron muy bien. Fue también en la institución pediátrica donde en 1993 se iniciaron los cursos formativos de educadores en diabetes en nuestro país y la educación en diabetes en diferentes modalidades se fortaleció e incluyó como parte del tratamiento por medio de la consulta, campamentos, sesiones de aula o individualizadas y talleres. La mayoría de los pacientes estaban en lo que se conocía como esquema de tratamiento convencional intensificado y las $\mathrm{HbA} 1 \mathrm{c}$ en promedio para todas las edades disminuyeron al $11 \%$. En esta década se confirmó una notable disminución en los casos incidentes de complicaciones crónicas, la mayoría leves detectadas por escrutinio. El grupo de pacientes menores de 10 años, mucho más dependientes de las decisiones tomadas por el cuidador primario informal (CPI), habitualmente ha sido aproximadamente el $30 \%$ de los casos. Con el fin de facilitar el proceso educativo para supervisión médica, en 1997 iniciaron los campamentos institucionales para niños pequeños y su CPI, generalmente la madre. En esta década la mayor disponibilidad de glucagón, generalmente por donativos, permitió el manejo óptimo de hipoglucemias severas ambulatorias.

En el 2000 se constituyó formalmente la subespecialidad de EP con reconocimiento universitario y llegó la época de los análogos de insulina rápida, que con su farmacocinética más estable permitieron ajustes más precisos y menos hipoglucemia. Su uso conjunto con la insulina basal NPH permitió un 
mejor manejo de la glucemia posprandial y cubrir mejor las elevaciones atribuibles a colaciones. En caso de disponibilidad limitada, su indicación fue prioritaria para población menor de 10 años, más susceptible a hipoglucemias y con mayor variabilidad glucémica. La disponibilidad de dispositivos o jeringas de 30 o $50 \mathrm{U}$ facilitaron el uso preciso de dosis de 1 o $0.5 \mathrm{U}$, aun sin la disponibilidad de diluyente (Tabla 2). En esta década fue evidente que una mayor proporción de madres que fungen como CPI laboraban ya fuera de casa y el rol tenía que ser más compartido. Las diferentes sociedades médicas establecieron metas de control glucémico más estrictas para pacientes pediátricos, gracias a la disponibilidad de análogos de insulina y avances tecnológicos en monitoreo glucémico y dispositivos de administración más precisos, en el entendido de que los mecanismos implicados en las complicaciones crónicas están presentes desde el diagnóstico. La HbA1c promedio era del $10.0 \%$ y las complicaciones agudas y crónicas disminuyeron considerablemente.

En el 2010 llegaron los análogos de insulina de segunda generación, principalmente de acción prolongada. El mayor costo de estas insulinas por su característica de patente ha limitado su utilización de acuerdo con presupuesto institucional o recursos personales. Junto con el monitoreo glucémico capilar estructurado o en la minoría de los casos continuo y el énfasis en la educación en diabetes y participación del paciente y su familia, las hipoglucemias se minimizaron y fue posible mejorar un poco más el control glucémico. Su limitación en la mezcla de dosis incrementó el número de aplicaciones y nos enfrentamos con problemas de adherencia. Sus concentraciones más estables se acompañaron de hiperglucemias atribuibles a colaciones escolares, previamente cubiertas por la insulina regular y/o NPH, por lo que en esos casos continuamos con el uso de insulina regular antes del desayuno, incluso junto con análogos de insulina rápida. Hay que señalar que el paciente pediátrico institucional con monitoreo capilar en la mayoría de los casos no realiza monitoreo ni cuenta con apoyo de personal de salud durante las horas de escuela y no necesariamente está capacitado para ajustes de insulina.
Tabla 2. Avances tecnológicos en insulinoterapia. Beneficios en el paciente pediátrico

\begin{tabular}{l} 
Menor variabilidad de la glucosa \\
Menor tiempo y severidad en disglucemia \\
\hline Objetivos de control semejantes a población adulta \\
\hline Mayor adherencia \\
\hline Ajustes más precisos \\
Menor invasividad y mejor adherencia \\
Ausencia de complicaciones crónicas en menores de 18 años
\end{tabular}

Ya que el paciente asume el costo de los insumos del monitoreo glucémico, la proporción de usuarios de monitores continuó siendo baja, aunque el monitoreo capilar estructurado se realizaba en aproximadamente el $60 \%$ de los casos. Esto, acompañado de correctas decisiones, mejoró los niveles promedio de $\mathrm{HbA} 1 \mathrm{c}$ al $9.5 \%$ en niños y adolescentes, que desciende al $8.0 \%$ en el grupo de prepúberes con gran impacto en la calidad de vida y pronóstico de nuestros pacientes.

\section{¿CÓMO ESTAMOS ACTUALMENTE?}

La mayoría de los pacientes pediátricos con DM1 son referidos al subespecialista, aunque no todas las instituciones pueden apoyar al paciente con su traslado. La mayoría de los pacientes conocidos por un servicio de EP continúan su manejo por subespecialistas, con apoyo en lugares más accesibles a su lu-

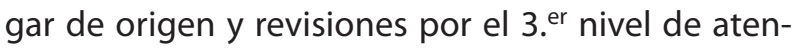
ción más espaciadas y por las condiciones de pandemia el equipo médico apoya con el incremento de asesoría a distancia para el paciente y su familia. De acuerdo con la institución, al paciente se le proporciona la consulta médica y estudios laboratorio requeridos a bajo costo, acorde a una categoría que considera nivel socioeconómico y acceso a otros servicios médicos. La dotación de insulina depende de la institución que puede incluirla sin costo siempre de acuerdo con el cuadro básico institucional y hospital específico y que significan un retraso importante de disponibilidad y está limitado a la dotación autorizada. En otras instituciones, el 
paciente adquiere los medicamentos por su cuenta, por lo que no está limitado a una dotación existente mas sí a los recursos propios. Los insumos como jeringas o plumas, tiras reactivas o monitores continuos de glucosa no son parte de dotación regular en prácticamente ninguna institución de salud, aun con protocolos de investigación, apoyo de la industria farmacéutica o donativos, por lo que la disponibilidad de información sobre parámetros de control más allá de la HbA1c es muy limitada. En general, se intenta que en el paciente pediátrico institucional la toma de estudios, la consulta y actividades educativas se programen en lo posible en un periodo de entre 24 y $48 \mathrm{~h}$ y la mayoría de los pacientes recibe un esquema de múltiples dosis, con uso de análogos en el 50-90\% de los casos, aunque el monitoreo glucémico capilar estructurado o continuo aún es insuficiente. Los pacientes pediátricos manejados en la institución se mantienen en general asintomáticos, con pocos eventos de hospitalización y control glucémico aceptable equiparable a otros países con las mismas condiciones. Desde hace más de dos décadas, prácticamente no se observan complicaciones crónicas ni muertes relacionadas con el descontrol glucémico en los servicios de EP del 3. er nivel de atención. Finalmente, en la transición al cuidado adulto, el paciente pediátrico manejado institucionalmente por el subespecialista hasta los 17-18 años de vida habitualmente cuenta con un buen grado de sensibilización y educación en el autocuidado de su enfermedad, siempre con opción de mejorar. Por lo que es importante que el endocrinólogo de adultos dé continuidad a la participación del paciente en el manejo de su enfermedad.

\section{EL PACIENTE ADULTO CON DIABETES MELLITUS TIPO 1 A NIVEL INSTITUCIONAL}

En el Instituto Nacional de Ciencias Médicas y Nutrición Salvador Zubirán (INCMNSZ), como en todas las instituciones del sector salud que no son dependientes del IMSS o del Instituto de Seguridad y Servicios Sociales de los Trabajadores del Estado
(ISSSTE), el acceso a la atención médica puede ser gratuita, pero como pacientes externos, ellos mismos deben aportar de sus propios recursos para insulina, medicamentos o monitoreo de la glucosa. La mayoría de los pacientes son de muy bajos recursos económicos y tienen limitantes importantes para llevar tratamientos intensivos, y la diabetes, como muchas enfermedades crónicas, condiciona un mayor empobrecimiento. Hoy en día la población del instituto ha cambiado y cada vez hay más pacientes de estratos socioeconómicos medios y con más recursos para lograr los objetivos terapéuticos. Por medio de trabajo social y con el apoyo de educadores en diabetes se trata de apoyar para que no haya ningún paciente atendido en la institución que no tenga acceso a la insulina, pero hasta la fecha el costo del cuidado de la diabetes recae principalmente en el paciente y por lo mismo para la gran mayoría de los pacientes con DM1 el costo, por ejemplo, de análogos de insulina los hace inaccesibles y son pocos los pacientes que pueden realizar monitoreo ambulatorio frecuente de la glucosa o tener un sensor para monitoreo continuo de glucosa.

El cambio en el modelo de atención de la diabetes se ha dado gradualmente, aunque el exceso en la carga asistencial, la falta de apoyo multidisciplinario en un inicio y las características propias de muchos pacientes dificultan su implementación ${ }^{12}$. Aproximadamente el $15 \%$ de la población atendida en la clínica de diabetes de INCMNSZ tiene diagnóstico de DM1 de inicio en la infancia o adolescencia, la mayoría con más de 10 años de evolución de la enfermedad.

En los años 60 y 70 la mayoría de los pacientes atendidos en el departamento eran adultos jóvenes de inicio en edad pediátrica y con complicaciones tardías de la enfermedad. Aunque hay un grupo de pacientes con inicio de la diabetes ya como adultos jóvenes y en los cuales la reserva de insulina habitualmente se va terminado en forma más gradual, con una luna de miel más prolongada y requerimientos bajos de insulina por muchos años. La cetoacidosis era un motivo frecuente de ingreso al hospital, así como la atención de complicaciones tardías de la enfermedad, incluyendo algunos pacientes con síndrome de Mauriac por el descontrol 
crónico grave con desnutrición y hepatomegalia. Desde esa época se tenía un manual pequeño para el paciente y se iniciaron cursos con duración de una semana, cuyo objetivo era ofrecer todas las herramientas y conocimientos requeridos para el cuidado cotidiano de la diabetes y para ayudar al paciente a aceptar su enfermedad con la participación del médico, enfermera y una licenciada en nutrición. La formación del médico incluía desde entonces un conocimiento amplio de nutriología para saber prescribir un plan de alimentación apropiado para cada paciente y motivar la realización de ejercicio. No había tiras reactivas para glucosa en sangre, ni medición de HbA1c y el monitoreo se realizaba con glucosurias fraccionadas cuando acudían a su consulta (no se podía hacer en forma ambulatoria). La mayoría de los pacientes era de muy bajos recursos y probablemente si hubiera forma de medirla la $\mathrm{HbA} 1 \mathrm{c}$ promedio habría sido mucho mayor al $10 \%$. El método de glucosurias en muestras fraccionadas prevaleció todavía en los años 80 no obstante las dificultades para recolectar la orina de las $24 \mathrm{~h}$ y guardarla en diferentes frascos ajustados al tiempo de recolección. Los pacientes muy descontrolados excretan 50 a $100 \mathrm{~g}$ de glucosa por día, por el contrario, pacientes con buen control metabólico deben excretar menos de 10 a $20 \mathrm{~g}$ de glucosa en orina de $24 \mathrm{~h}^{13}$. Además de la gran incomodidad que representaba para el paciente cargar los frascos, la mayoría pacientes de bajos recursos económicos y/o que viajaban desde sus localidades de origen, lo que significaba que sus muestras no fueran representativas de un día habitual.

A mediados de los años 70 se inicia el monitoreo ambulatorio de glucemias capilares en tiempo real por comparación visual con una escala de colores, con apoyo de glucómetros posteriormente y medición de hemoglobina glucosilada a principios de los 80. Los primeros reportes de $\mathrm{HbA} 1 \mathrm{c}$ de los diferentes centros hospitalarios del país de sus pacientes con DM1 eran alarmantes, con cifras promedio de $\mathrm{HbA} 1 \mathrm{c}$ del 12 al $15 \%{ }^{14}$. El manejo del paciente con DM1 era muy diferente al que se puede ofrecer hoy en día, gradualmente se fue agregando un esquema fijo de NPH combinado con insulina rápida dos veces al día (antes no era raro encontrar pacientes que solo se aplicaban una dosis de NPH al día) y en aquellos pacientes que podían ya comprar un glucómetro y tiras reactivas se ofrecía un esquema más intensivo con ajustes en los bolos de insulina rápida con base en sus lecturas preprandiales y consumo de carbohidratos y agregando una tercera dosis de insulina rápida a mediodía.

La introducción de jeringas desechables, aunque aún sin la fineza de las agujas actualmente disponibles y en 1982 de la insulina humana por tecnología recombinante, favoreció una mejor adherencia, menos reacciones de alergia local o lipodistrofia, y contribuyó a mejorar el control glucémico. Hasta finales de los 80 todavía los pacientes seguían Ilegando, cargando sus frascos con orina para medir las glucosurias fraccionadas. De ser posible, insistíamos en realizar monitoreo de la glucosa capilar preprandial por tres días (igualmente, con tiras cortadas en sentido vertical a la mitad para que duplicaran el número de tiras y usando un método visual por cambio de colores). Con esta información se hacían ajustes en su esquema de tratamiento.

Aún la gran mayoría de los pacientes utilizaban insulina bovina y el esquema tradicional era la mezcla de NPH más rápida en una misma jeringa antes del desayuno y de la cena (en muy pocos pacientes se utilizaban tres dosis); el promedio de $\mathrm{HbA} 1 \mathrm{c}$ en nuestros pacientes con DM1, la mayoría provenientes de medio socioeconómico bajo, no era menor del $9-10 \%$ y desafortunadamente éramos testigos de la historia natural de la enfermedad con una prevalencia muy elevada de todo tipo de complicaciones derivadas de la enfermedad. Hay pacientes que por la pobreza en que vivían no podían comprar su insulina (se intentaba tener siempre insulina disponible para ellos y el apoyo por trabajo social). Algunos no podían refrigerar su insulina y la guardaban en el lugar más frío de la casa e incluso algunos campesinos en el riachuelo que pasaba cerca de sus hogares. En este tipo de pacientes, el objetivo es que sobrevivieran el mayor número de años posible con la mejor calidad de vida y sin ingresar frecuentemente al servicio de urgencias con episodios de cetoacidosis. Una causa frecuente de internamiento en el instituto era y sigue siendo el pie diabético asociado a la patología de la pobreza, donde el paciente no tiene un calzado apropiado ni los cuidados que requiere para prevenir lesiones en 
sus pies afectados por una neuropatía severa y/o daño vascular. No era raro que el paciente después de 5-6 semanas de internamiento y «salvar» el pie, regresara a casa, se olvidara del reposo y los cuidados por su imperiosa necesidad de trabajar. Este paciente con frecuencia regresaba a las pocas semanas, con un nuevo proceso séptico en el pie para ser amputado.

Los análogos de insulina rápida se empezaron a utilizar en los 90, con un costo mucho mayor y poco accesible a pacientes de nuestra institución. Como medida de comparación, ya se empezaban a utilizar las estatinas, pero pocos pacientes tenían acceso a estas por razones económicas.

El año 1993 fue muy importante en la historia del manejo del paciente con DM1. Se presentaron los datos del $D C C T^{1,2}$, que demostró el beneficio de un control intensivo para prevenir complicaciones tardías de la enfermedad, aunque incrementando, como es de esperarse, el riesgo de hipoglucemia (uno de cada tres pacientes en el grupo intensivo presentó hipoglucemias severas con pérdida del conocimiento), esto no obstante que se excluyeron para este estudio pacientes con historia de hipoglucemias frecuentes. A partir de ese momento se refuerza la importancia de un manejo intensivo y de lograr cifras de HbA1c más bajas, la principal barrera es la falta de recursos de la mayoría de los pacientes. Sin monitoreo, el riesgo de hipoglucemias severas es mucho mayor. No obstante, las cifras de $\mathrm{HbA1c}$ siguen descendiendo y el médico adquiere un papel cada vez más importante como educador y motivador y se refuerza la importancia de un apoyo multidisciplinario.

La mayoría de los pacientes con DM1 que ingresaban a la institución generalmente llegaban después de los 25 años y muchos ya por complicaciones tardías de la enfermedad. En un estudio que se hizo hace 20 años en colaboración con el INP y el Centro Médico Nacional Siglo XXI, encontramos que de 209 pacientes egresados por mayoría de edad, el $70 \%$ no llegaban a los centros especializados de referencia y continuaba su manejo en el primer nivel de atención, no por el médico especialista y en el mejor de los casos sin dejar el mismo tratamiento que tenían al egresar de la institución ${ }^{15}$. Lo anterior resaltaba la necesidad de reforzar el proceso de transición, que generalmente se dificulta por aspectos administrativos y las dificultades de continuar con seguimiento médico para el adulto joven, cuando sus prioridades son trabajar y resolver sus necesidades económicas. En el INCMNSZ en el 2008 la DM2 de inicio temprano constituía el $27.8 \%$ de todos los pacientes con diabetes que se atendían en la consulta externa y alrededor del $16 \%$ de toda la consulta de diabetes la constituían pacientes con DM1.

En pacientes con DM1 de muy bajos recursos se utilizaban tres dosis de NPH (antes del desayuno, comida y antes de acostarse), con lo que los picos de insulina sustituyen de alguna manera la necesidad de rápida antes de la comida y de la cena. En pacientes con buena adherencia al plan de alimentación y actividad física con este esquema logramos llevarlos a una $\mathrm{HbA} 1 \mathrm{c}$ alrededor del $8.5 \%$, que son resultados muy satisfactorios. Hay que recordar que el DCCT se continuó como el estudio $\mathrm{EDIC}^{16}$ que reflejó la evolución de los pacientes con su manejo intensivo habitual, en estos la media de $\mathrm{HbA} 1 \mathrm{c}$ alcanzada fue del $8.0 \%$. En otros pacientes, sobre todo adultos jóvenes, pero con poca posibilidad de automonitoreo, empleamos la mezcla de insulinas $\mathrm{NPH}$ y rápida o ultrarrápida dos o tres veces al día como alternativa, la motivación del médico y del paciente es fundamental, ya que se requiere de mucho trabajo, tiempo y retroalimentación frecuente.

El manejo del paciente con DM1 se transformó, se desarrolló una clínica específica de diabetes tipo 1 en la cual el manejo intensivo se implementa en la mayoría de los pacientes con uso de análogos de insulina en la mayoría y uso de monitoreo continuo de glucosa en una proporción de pacientes. Además, existe una clínica de microinfusoras para algunos pacientes. El uso de análogos de insulina y de la tecnología en diabetes está siempre limitado por el costo que representan para los pacientes. Se dio más énfasis a la educación con más participación de educadores en diabetes y al apoyo multidisciplinario en forma temprana enfocado a lograr los objetivos terapéuticos y prevención de complicaciones. Se reconoce la gran importancia del apoyo emocional y detectar problemas psiquiátricos y con ayuda del departamento de psiquiatría se da atención a estos padecimientos. Aun con todos estos avances, 
el promedio de $\mathrm{HbA} 1 \mathrm{c}$ es de alrededor del $8.5 \%$ y un porcentaje significativo de pacientes presenta complicaciones crónicas, en parte debido al largo tiempo de evolución de su enfermedad. Se hace mucho énfasis en la detección y tratamiento temprano de estas y en prevenir sus complicaciones con el apoyo de la clínica del pie diabético, la referencia temprana a oftalmología y el manejo agresivo de los factores de riesgo cardiovascular.

No podemos incluir aquí la información de lo que ocurre en otras instituciones de salud, pero es evidente que dependerá de la preparación y motivación del médico, la carga asistencial (se requiere tiempo para motivar y educar), el acceso a apoyo multidisciplinario y de los recursos de que dispongan los pacientes para poder lograr un manejo intensivo de la diabetes (es muy diferente si el paciente tiene acceso a una institución donde se le proporciona todo el tratamiento, incluyendo métodos de automonitoreo). En algunos escenarios las cifras promedio de HbA1c podrán estar cercanas al $8.0 \%$, en otros continuarán arriba del $10.0 \%$ como antes. Muchos pacientes, como comentamos, ya ingresan con complicaciones tardías graves de la enfermedad y es poco lo que podemos hacer para modificar la historia natural de la enfermedad, excepto atender de la mejor manera estas complicaciones.

\section{LA EXPERIENCIA EN LA PRÁCTICA PRIVADA}

En la medicina privada, las estrategias y limitaciones de los esquemas y dispositivos han tenido la misma evolución, aunque existe mayor disponibilidad de recursos y acceso más temprano a nuevas insulinas y tecnología en comparación con la medicina institucional, claro está dependerá del tipo de práctica privada y los recursos del paciente. Aproximadamente el $20 \%$ de los pacientes recibe atención mixta, con apoyo institucional para medicamentos y laboratorio y seguimiento una o hasta dos veces por año en la práctica privada. Además de la posibilidad de elección del equipo de salud geográficamente más accesible, es habitual la retroalimentación a distancia y los motivos agudos de hospitalización son poco frecuentes. La mayoría de los pacientes utilizan monitoreo continuo de glucosa y esquema de múltiples dosis (hasta 4-5 aplicaciones de insulina al día en algunos casos), algunos médicos (muy pocos) tienen hasta un 30 a $40 \%$ de sus pacientes con microinfusoras que en muchos casos pueden ser cubiertas por seguros de gastos médicos. Es factible lograr cifras de $\mathrm{HbA} 1 \mathrm{c}$ en rangos del 6.5 a 7\%. Comparado con el manejo institucional de 3 er $^{\text {er nivel, }}$ todo lo anterior puede significar mayor adherencia, flexibilidad y asesoría con diferencias de $\mathrm{HbA} 1 \mathrm{c}$ de entre el 1 y $1.5 \%$ y parámetros más allá de la $\mathrm{HbA} 1 \mathrm{C}$ (tiempo en rango, variabilidad, tiempo en hipoglucemia e hiperglucemia disponibles más cercanos a lo deseable). Aunque no contamos con estadísticas precisas, se espera una aparición más tardía y menos severa de complicaciones, a pesar del mayor riesgo de hipoglucemias, que afortunadamente se ha visto reducido en los últimos años gracias al uso de sensores para monitoreo continuo de la glucosa. El paciente adulto joven que ya es todo un experto en el manejo de su diabetes y por lo demás sano, muchas veces omite sus visitas al médico por temporadas (si acaso se hace estudios de HbA1c cada 6-12 meses) y regresa cuando tiene alguna enfermedad intercurrente y requiere de nuestra atención.

\section{LAS BARRERAS EN EL TRATAMIENTO DE LA DIABETES TIPO 1}

La educación es una parte muy importante del tratamiento y se inició a partir del momento que se empezó a utilizar insulina en la práctica clínica, esto es, hace 100 años. El rol de educador inicialmente lo tenía principalmente la enfermera; hoy en día tanto el médico como la enfermera, el licenciado en nutrición y otros especialistas deben estar capacitados para dar educación en diabetes y así ofrecer las herramientas para valorar y comprender la utilidad del tratamiento, tomar decisiones día a día y prevenir y tratar complicaciones agudas y crónicas de la enfermedad. Hay muchos factores que dificultan la adherencia al tratamiento o impiden lograr un manejo intensivo, entre ellas están la capacidad real de comprensión (inteligencia), los mecanismos de adaptación a la 
enfermedad (personalidad) y la falta de información, problemas familiares, económicos y culturales, ansiedad, depresión o incluso trastornos de la conducta alimentaria. No se puede pretender un tratamiento intensivo en pacientes poco motivados, con pobre adherencia al tratamiento y mínimas conductas de autocuidado, aquellos con poco apoyo familiar y mínimos recursos económicos ${ }^{17}$.

El manejo intensivo de la diabetes conlleva un mayor riesgo de hipoglucemia, limitante principal para lograr los objetivos terapéuticos en muchos de nuestros pacientes. En el DCCT la prevalencia anual de hipoglucemia severa fue del $36 \%$, cuando se publicó el DCCT no había análogos de insulina ni sensores para monitoreo continuo, desafortunadamente la mayoría de los pacientes con DM1 en México no tiene acceso a muchos de los adelantos tecnológicos por su costo elevado.

La hipoglucemia cuando es severa y frecuente genera inseguridad, miedo, pobre adherencia, aumento de peso y menor calidad de vida, con efecto negativo en las relaciones interpersonales y con implicaciones en el trabajo. Es por ello que todo programa de manejo intensivo requiere forzosamente ofrecer educación y alternativas de cómo prevenir y tratar estos episodios. La parte emocional es muy importante en toda enfermedad crónica y en particular en la $\mathrm{DM} 1{ }^{18}$, lo primero es ayudar a que el paciente y la familia acepten la enfermedad y aprendan a vivir con ella. Para muchos padres es muy difícil iniciar la rutina diaria del cuidado de la diabetes; para unos, la dificultad principal radica en aplicar las inyecciones y realizar los piquetes en los dedos, para otros, modificar el plan de alimentación; otros más tienen problemas para controlar sus miedos, particularmente a las complicaciones o hipoglucemias que pudiesen presentarse sea en la escuela o mientras duermen. La adolescencia es un periodo crítico en el cuidado de la diabetes, en el cual es difícil mantener los objetivos terapéuticos. En este periodo el adolescente puede cuestionar seriamente su imagen corporal y su autoestima, suele sentir que la enfermedad y los requerimientos de su manejo amenazan su posibilidad de hacer planes para el futuro y de llevarlos a cabo. Estos y otros factores pueden dar lugar a que el adolescente se rebele contra la diabetes y descuide aspectos del programa de tratamiento y en ocasiones, se omitan dosis de insulina.
La asociación con sobrepeso u obesidad y antecedentes de DM2 en la familia agrega el factor de resistencia a la insulina y malos hábitos de alimentación y ejercicio que dificultan en forma muy importante el logro de los objetivos terapéuticos y se asocian a mayor riesgo de complicaciones.

La parte económica es primordial en la adherencia al tratamiento en las enfermedades crónicas; en pacientes de estrato socioeconómico bajo, si el sistema de salud no ofrece tratamiento y seguimiento médico gratuito, el costo de los medicamento y métodos de automonitoreo lo empobrece más y le impide cubrir sus otras necesidades del día a día ${ }^{19}$. Al no seguir un tratamiento en forma adecuada, tendrán mucho mayor riesgo de desarrollar complicaciones, y requerir mayores gastos y atención médica con el paso de los años.

\section{REFLEXIONES FINALES}

En la últimas tres décadas y derivado de los grandes avances tecnológicos y las evidencias del importante beneficio de lograr los objetivos terapéuticos hemos observado cambios muy importantes en el manejo del paciente con DM1 que se traducen en reducciones muy significativas de $\mathrm{HbA} 1 \mathrm{c}$ y una menor incidencia de complicaciones de la enfermedad. Si bien la tecnología ha permitido un avance considerable en el manejo óptimo de la DM1 a todas las edades, esto no sería posible sin la educación en diabetes, el manejo integral de la enfermedad en sus aspectos biopsicosociales y el compromiso de todo el equipo de salud. Las limitaciones educativas, económicas y de acceso a la salud es una limitante aún muy importante en nuestro país para alcanzar los objetivos terapéuticos.

\section{FINANCIAMIENTO}

La presente investigación no ha recibido ninguna beca específica de agencias de los sectores públicos, comercial, o con ánimo de lucro. 


\section{CONFLICTO DE INTERESES}

Los autores declaran no tener ningún conflicto de intereses.

\section{RESPONSABILIDADES ÉTICAS}

Protección de personas y animales. Los autores declaran que para esta investigación no se han realizado experimentos en seres humanos ni en animales.

Confidencialidad de los datos. Los autores declaran que en este artículo no aparecen datos de pacientes.

Derecho a la privacidad y consentimiento informado. Los autores declaran que en este artículo no aparecen datos de pacientes.

\section{BIBLIOGRAFÍA}

1. Nathan DM. Realizing the long-term promise of insulin therapy: the DCCT/EDIC study. Diabetologia. 2021;64(5):1049-58.

2. The Diabetes Control and Complications Trial Research Group. The effect of intensive treatment of diabetes on the development and progression of long-term complications in insulin-dependent diabetes mellitus. $\mathrm{N}$ Engl J Med. 1993;329:977-86.

3. Faradji RN, Valenzuela-Lara $M$, Vidrio-Velázquez $M$, Yepez-Rodríguez $A E$, González-Galvez G, Sainz de la Maza-Viadero ME; Members of RENACEDDT1 Research Group in alphabetical order. RENACED-DT1: A National Type 1 Diabetes Registry Initiative in Mexico. Salud Publica Mex. 2020;62(3):232-4.
4. Haller MJ, Atkinson MA, Schatz D. Type 1 diabetes mellitus: etiology, presentation, and management. Pediatr Clin North Am. 2005;52(6):1553-78.

5. Gómez-Díaz RA, Pérez-Pérez G, Hernández-Cuesta IT, Rodríguez-García J del C, Guerrero-López R, Aguilar-Salinas CA, et al. Incidence of type 1 diabetes in Mexico: data from an institutional register 2000-2010. Diabetes Care. 2012;35(11):e77.

6. Díaz-Valencia $P A$, Bougnères $P$, Valleron $A J$. Global epidemiology of type 1 diabetes in young adults and adults: a systematic review. BMC Public Health. 2015;15:255.

7. Aguilar-Salinas CA, Rojas R, Gómez-Pérez FJ, García E, Valles V, Ríos-Torres $J M$, et al. Prevalence and characteristics of early-onset type 2 diabetes in Mexico. Am J Med. 2002;113(7):569-74.

8. Lerman I, Granados J, Aguilar-Salinas C, Lobato M, Villa RA, Velasco ML et al. Baja prevalencia de autoinmunidad (anticuerpos antiGAD +) en pacientes adultos con diabetes tipo 2 de inicio temprano. Rev Endocrinol Nutr. 2010;18:170-5.

9. Umpierrez G, Korytkowski M. Diabetic emergencies: ketoacidosis, hyperglycaemic hyperosmolar state and hypoglycaemia. Nat Rev Endocrinol. 2016;12(4):222-32.

10. Chiquete E, Ochoa Guzmán A, Ramírez Mejía Y, Cantú Brito C. Hospitalización por complicaciones agudas de la diabetes mellitus en el sistema sanitario público mexicano durante el año 2012. Rev Endocrinol Nutr. 2014:1:152-7.

11. Lipton R, Good G, Mikhailov T, Freels S, Donoghue E. Ethnic differences in mortality from insulin-dependent diabetes mellitus among people less than 25 years of age. Pediatrics. 1999;103(5 Pt 1):952-6.

12. Lerman I. La atención del paciente más allá del primer nivel de atención. Salud Publica Mex. 2007;49:E99-E103.

13. Feldman JM, Lebovitz FL. Tests for glucosuria. An analysis of factors that cause misleading results. Diabetes. 1973;22(2):115-21.

14. Bravo Ríos EL, Cardoso Saldaña G, Torres Tamayo M, Lerman Garber I, Zamora González J, García Bulnes G, et al. Microalbuminuria y factores de riesgo macrovascular en niños diabéticos insulino-dependientes. Rev Invest Clin. 1996:48(1):19-25.

15. Lerman Gl, Barrón C, Calzada LR, Mercado M, Vidal R, Quintana S, et al. Transition from adolescence to young adulthood in diabetes care. Diabetes. 2001;50(2):393.

16. Epidemiology of Diabetes Interventions and Complications (EDIC) Research Group. Epidemiology of Diabetes Interventions and Complications (EDIC). Design, implementation, and preliminary results of a longterm follow-up of the Diabetes Control and Complications Trial cohort. Diabetes Care. 1999;22(1):99-111.

17. Lerman I. Adherence to treatment: the key for avoiding long-term complications of diabetes. Arch Med Res. 2005;36(3):300-6.

18. Delamater AM, de Wit M, McDarby V, Malik JA, Hilliard ME, Northam E, et al. ISPAD Clinical Practice Consensus Guidelines 2018: Psychological care of children and adolescents with type 1 diabetes. Pediatr Diabetes. 2018;19(Suppl 27):237-49.

19. Altamirano-Bustamante $N$, Islas-Ortega L, Robles-Valdés C, GarduñoEspinosa J, Morales-Cisneros G, Valderrama A, et al. Economic family burden of metabolic control in children and adolescents with type 1 diabetes mellitus. J Pediatr Endocrinol Metab. 2008;21(12):1163-8. 